\title{
Ephemeroptera, Plecoptera, and Trichoptera on Isle Royale National Park, USA, compared to mainland species pool and size distribution
}

\author{
R. Edward DeWalt', Eric J. South ${ }^{2}$ \\ I University of Illinois, Prairie Research Institute, Illinois Natural History Survey, 1816 S Oak St., Cham- \\ paign, IL 61820, USA 2 University of Illinois at Urbana-Champaign, Department of Entomology, 320 Mor- \\ rill Hall, 505 S. Goodwin Ave., Urbana, IL 61801, USA
}

Corresponding author: R. Edward DeWalt (dewalt@illinois.edu)

Academic editor: B. Price | Received 4 September 2015 | Accepted 13 October 2015 | Published 5 November 2015

http://zoobank.org/37B79D4A-18D0-4E73-9B85-616F2254FC91

Citation: DeWalt RE, South EJ (2015) Ephemeroptera, Plecoptera, and Trichoptera on Isle Royale National Park, USA, compared to mainland species pool and size distribution. ZooKeys 532: 137-158. doi: 10.3897/zookeys.532.6478

\begin{abstract}
Extensive sampling for aquatic insects was conducted in the orders Ephemeroptera (mayflies), Plecoptera (stoneflies), and Trichoptera (caddisflies) (EPT) of Isle Royale National Park (ISRO), Michigan, United States of America, during summer 2013. The island was ice covered until 8,000 to 10,000 years ago and is isolated by $22-70 \mathrm{~km}$ distance from the mainland. Two hypotheses were examined: that ISRO EPT richness would be much reduced from the mainland, and that the species colonizing ISRO would be of smaller size than mainland, adults presumably using updrafts to bridge the distance from mainland sources. Data sets were developed for known mainland EPT species and size for those species. The first hypothesis was confirmed with the mainland species pool consisting of 417 EPT, while ISRO is known to support 73 species. Richness of EPT is directly related to the number of specimens examined. Small streams supported five EPT species, while 15-25 species were found in larger streams. Lakeshores had intermediate diversity. The second hypothesis was substantiated for stoneflies, but not for mayflies or caddisflies. Stoneflies apparently are poorer fliers than either of the other two orders.
\end{abstract}

\section{Keywords}

Isle Royale National Park, Ephemeroptera, Plecoptera, Trichoptera, adult size, regional species pool 


\section{Introduction}

Isle Royale National Park (ISRO) is an archipelago of islands located in cold, oligotrophic Lake Superior, Michigan, United States of America (USA). The main island is $72 \mathrm{~km}$ long and $14 \mathrm{~km}$ wide at its greatest dimensions (Kraft et al. 2010). It is presumed that all macroscopic life was eliminated from the island during the Wisconsinan glacial episodes. The region has been ice free for as much as 10,000 yr. Life repopulated by various means from mainland sources, a distance of 20-22 km from Minnesota, USA and Ontario, Canada or $70 \mathrm{~km}$ from the Keweenaw Peninsula of Michigan (Fig. 1). The entire park was assessed for natural resource condition within the past decade and much of what is known about the island and its fauna and flora is contained within Kraft et al. (2010).

Little is known of the aquatic insects inhabiting the shores, streams, and lakes of ISRO (Bick et al. 1985). At least three ecological studies including aquatic insects have been conducted, but these involved sampling of larvae and genus level identification only, representing government literature and university theses that have not been published (Bowden 1981, Johnson 1980, Toczydlowski et al. 1979). A relatively few specimens of aquatic insects are known from regional museums (DeWalt unpubl. data).

The isolated nature of ISRO and lack of roads on the island make it logistically difficult to inventory. The island may be reached by ferry, personal watercraft, or by plane. It is undeveloped with the exception of a few locations, Windigo in the southwest and Rock Harbor in the northeast (Fig. 1). Waterbodies must then be accessed by foot or by Park Service boat. Few systematic entomologists have visited ISRO due to the expected depauperate nature of the fauna, though some species that currently live on the island will be of interest since they represent relict populations at the southernmost edge of their range. Another reason for studying aquatic insects at ISRO is to understand which species are capable of colonizing the island. Important questions include what species traits allowed them to colonize successfully, and potentially what sources and routes were involved in the colonization.

Ephemeroptera (mayflies), Plecoptera (stoneflies), and Trichoptera (caddisflies) (EPT species) are environmentally sensitive aquatic insects that are routinely used in monitoring of water quality. Their taxonomy and distribution are relatively well known in the Midwest (Burks 1953, DeWalt et al. 2005, DeWalt et al. 2012, DeWalt and Grubbs 2011, Frison 1935, Grubbs et al. 2013, Houghton 2012, Randolph and McCafferty 1998, Ross 1944). This makes EPT an appropriate target for inventories on ISRO.

The EPT species currently living on ISRO most certainly arrived through one or more of several means: direct flight, drifting with debris, as stowaways on boats, or were already present in the lake. Sources of colonization are streams and lakes along the shoreline of Lake Superior in Michigan, Minnesota, Ontario, and Wisconsin. We are assuming that most species would have flown to the island from mainland shoreline sources and that smaller insect species would arrive and establish in greater frequency than larger ones.

Recent work by DeWalt and colleagues has created a 200,000 record EPT specimen dataset within a seven state area of the Midwest, USA. These data have recently 


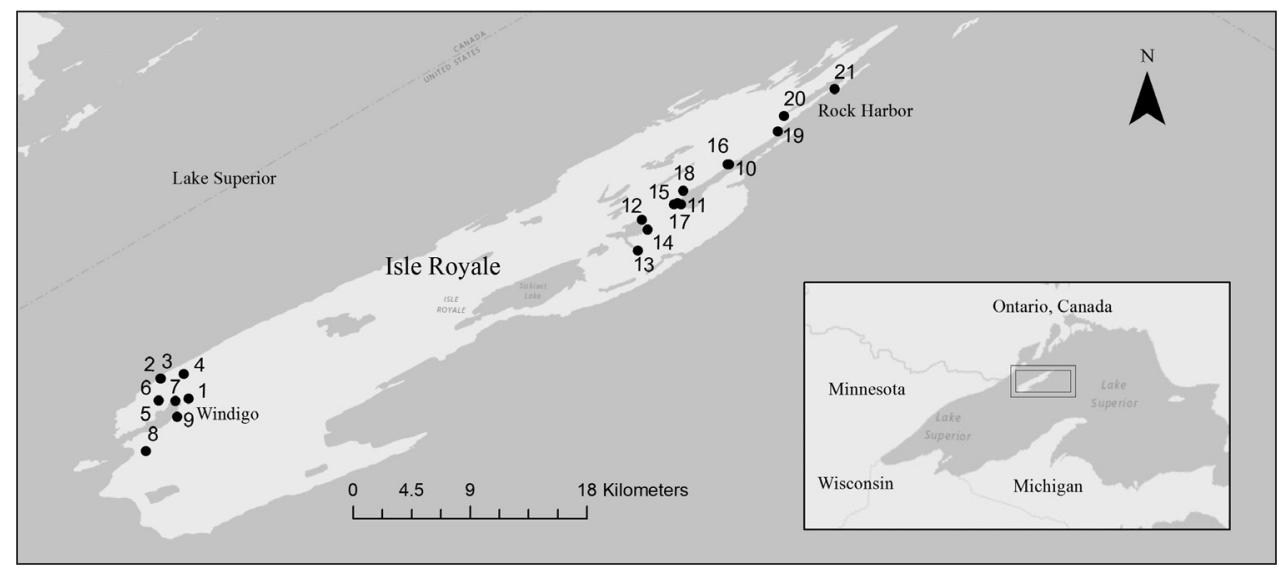

Figure I. Sampling locations on Isle Royale National Park, Michigan, USA, during 2013. Points and numbers indicate sample locations as defined in Table 1.

been used to model the historical distributions of stoneflies (Cao et al. 2013) and through the $21^{\text {st }}$ century (DeWalt, unpubl. data). This data set, some recently published records (Houghton 2012), and unpublished data (Klubertanz, pers. comm.) may be used as a tool to build a regional species pool for comparison with ISRO.

The results of our effort to document the EPT species inhabiting the main island of ISRO during 2013 are presented. We hypothesize that the number of EPT species on ISRO is less than that found on the mainland surrounding Lake Superior. In addition, we hypothesize that the size of adult EPT species present on ISRO is smaller than that found on the mainland, suggesting that smaller species are more likely to colonize the island from mainland sources, presumably using prevailing winds.

\section{Methods}

Inventory. Inventory of EPT taxa took place over two four-day forays in June and July 2013 (Table 1). Two areas of the island were investigated near access points for ferry service. Our June efforts were concentrated on Lake Superior shorelines and small streams near Windigo in southwestern ISRO (sites 1-9, Table 1, Fig. 1). July efforts concentrated on the same habitats from Rock Harbor in northeastern ISRO to the west end of Moskey Basin (sites 10-21).

Sampling of EPT was conducted in the same manner at each site and continued until no apparently novel taxa were found at a site. Collection of adults was prioritized since species level identification is most straightforward in this life stage. One of the most useful sampling devises for adult EPT ISRO was the beating sheet. The sheet was placed under vegetation at streamside or lakeside and adults dislodged to the sheet. This method was particularly effective when air temperatures were cool, limiting flight of insects after disturbance. Warmer conditions necessitated the use of an aerial 


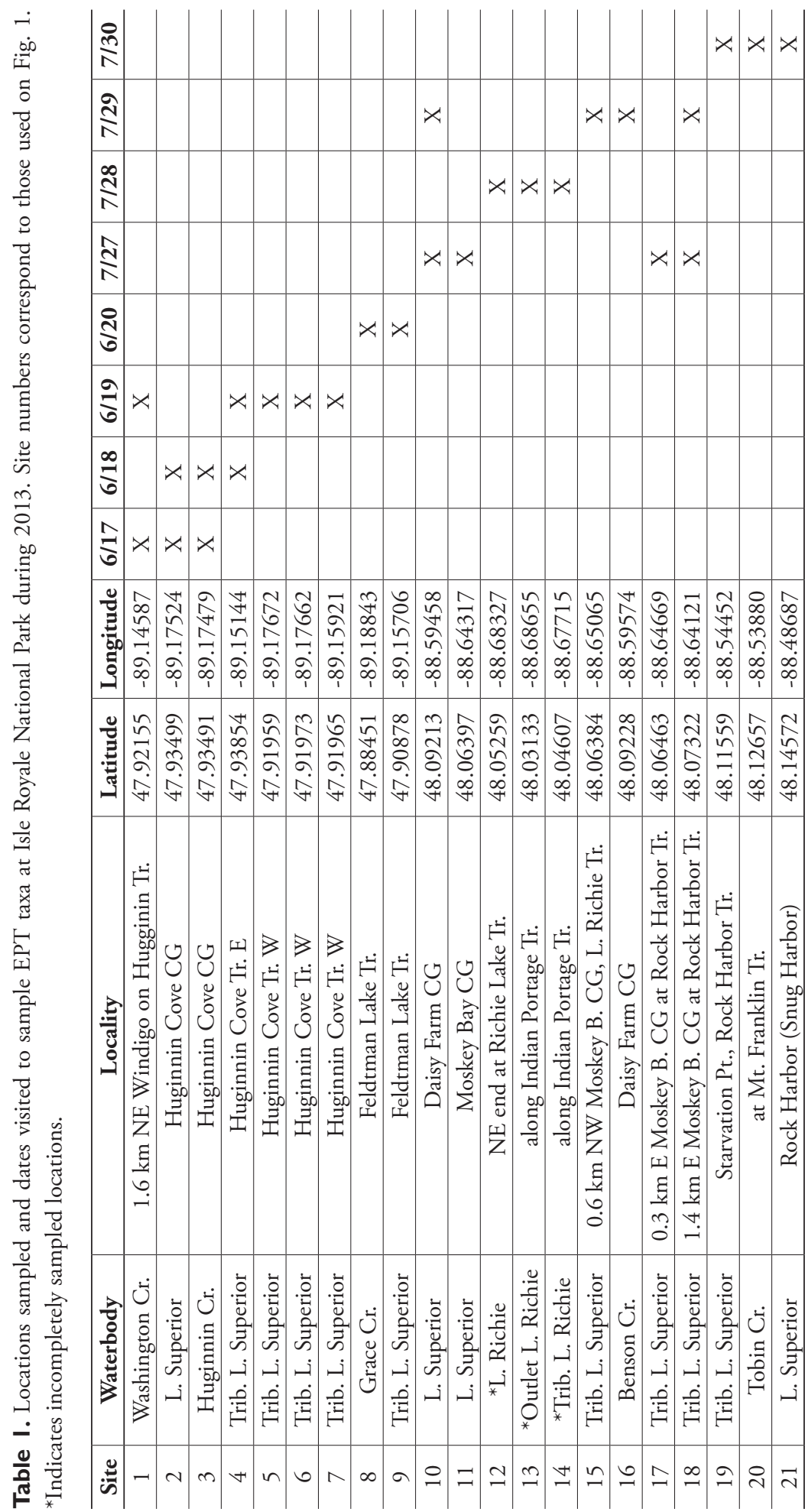


sweepnet. Immature EPT were collected using a rectangular dipnet and by handpicking from substrates. The accumulated debris were examined using a white plastic tray and stream water. All EPT specimens were fixed in 95\% EtOH. Non-target taxa were released after sorting. The use of ultraviolet light traps was not possible during either of the two visits due to the low early evening air temperatures.

Specimens were identified to the lowest possible taxonomic level, using current literature, and accessioned into the INHS Insect Collection (INHS-IC). These data are available from the INHS-IC database (http://inhsinsectcollection.speciesfile.org/ InsectCollection.aspx). Raw specimen data in the form of an Excel comma delimited file are attached as supplementary data.

The relationship between EPT species richness and the number of specimens collected per site was investigated using simple linear regression. This analysis was conducted on untransformed data using VassarStats (Lowry 2015) an internet based statistical package. Data from three samples (sites 12-14 of Table 1) were excluded from this analysis because they were considered incomplete.

Comparison of ISRO EPT to Mainland. The mainland list of EPT species was compiled from specimen records whose locations included the Lake Superior border counties of Michigan, Minnesota, and Wisconsin and streams that drained into Lake Superior from Ontario west of $-84.3^{\circ}$ longitude and south of a line delimited by $49.3^{\circ}$ latitude. Specimen data were pulled from the INHS-IC database, several other databases compiled by the senior author from 25 region museums, a regional treatment of mayflies (Randolph and McCafferty 1998), additional mayfly records (T. Klubertanz unpubl. data), and other recent literature (Houghton 2012, Blahnik and Holzenthal 2014, Sun and McCafferty 2008). Some of these data are unpublished, so the species list for the mainland is withheld at the owner's request. The ISRO list was compared directly to the mainland list.

Size of ISRO EPT Species Versus Mainland. Size of specimens was gathered from the literature, often from original species descriptions. Most useful was the Biodiversity Heritage Library, which has made access to older literature efficient. The measure of size varied greatly between sources. Forewing length was preferred, but often body length was the only measure presented. In some of the oldest literature (e.g., Walker 1852), measurements were provided in "lines". There is no accepted scale for conversion of lines to $\mathrm{mm}$, but a conversion of British lines to $2.12 \mathrm{~mm}$ has been offered through http://www.convertunits.com/from/line/to/mm. We have applied this conversion to all line measurements and the resultant sizes agree with congeners measured in $\mathrm{mm}$. For all but the largest of EPT species, body length appeared to be a suitable approximation of forewing length. In some instances, no adult measures were available, so length of mature larvae was recorded or measures from species in the same genus were used. Ranges of sizes were often presented in literature sources and were recorded as both minimum and maximum size. Admittedly, some error exists in the sizes recorded, but this appears to be the best that can be done without actually measuring replicates of several hundred species. Literature sources and the type of measure were recorded for all species. Those who wish to use the data set may request a copy from the senior author. 
Since we were only assured of a minimum size across the entire data set, this was the measure used for comparative purposes. Both integer and decimal values were present in the literature, so all were converted to the integer form of the value to simplify analysis. Frequency histograms with size classes from 1 to $34 \mathrm{~mm}$ were compared for mainland and ISRO species. A Kruskal-Wallis $\mathrm{k}=3$ analysis of ranked data was conducted to compare sizes of orders of EPT on the mainland and on the ISRO (Sokal and Rohlf 1981). In addition, a Mann-Whitney U-test was conducted on mainland versus ISRO adult size for each order (Sokal and Rohlf 1981). All tests of significance were run using Lowry (2015).

\section{Results}

ISRO EPT Richness and Comparison to Mainland. Twenty-nine samples were collected from ISRO during 2013, representing 21 locations from opposite ends of the island (Fig. 1, Table 1). These samples produced 983 specimens representing 73 species of EPT (Table 2). The vast majority of EPT species were caddisflies, contributing 42 of the 73 species reported. Mayflies contributed 22 species, while stoneflies contributed only nine. Site EPT richness varied dramatically (Fig. 2). The EPT species richness for completely sampled sites was a linear function of the number of individuals found at the site (simple linear regression, $\mathrm{R}^{2}=0.45, \mathrm{p}=0.002, \mathrm{n}=18$, Fig. 3). Washington Creek departed greatly from the line-of-best fit (Site 1 of Table 1, Fig. 3). This $5 \mathrm{~m}$ wide trout stream is much more diverse compared to other streams sampled during this project, supporting $25 \mathrm{EPT}$ species from a relatively modest number of specimens. The similarly sized Grace Creek (Site 8, Fig. 2) produced only 15 EPT species. Other relatively diverse sites were Lake Superior shorelines at Huginnin Cove (Site 2), Daisy Farm Campground (Site 10), and at Moskey Bay Campground (Site 11). Benson Creek (Site 16 of Table 1) under performed versus predicted richness. This $2 \mathrm{~m}$ wide stream produced just five EPT species including two mayflies, two stoneflies, and one caddisfly (Table 2, Fig. 2). Mainland richness was much higher than that found on ISRO, confirming our hypothesis. This trend held for each order sampled, with 417 EPT species being recorded from mainland specimen and literature sources (Fig. 4).

Species richness was predictable in relation to waterbody type and stream size. Streams 1-2 m wide supported a limited EPT fauna, averaging 5.3 species with narrow variability (Fig. 5). Larger streams supported many more species with much higher variation. Lake Superior shorelines, including areas open to the fetch of the lake and those in large protected bays, produced on average $10 \mathrm{EPT}$ species, with relatively low variability.

Most EPT species found on ISRO were rarely encountered, 51 of them being present in only one or two samples of 26 complete samples (Fig. 6). A relatively few species may be considered common on ISRO since they were found in $>4$ samples. Among these were five species: the caddisfly Neophylax concinnus McLachlan, 1871 and the stoneflies Amphinemura palmeni (Koponen, 1917), Leuctra ferruginea (Walker, 1852), Nemoura trispinosa Claassen, 1923, and Haploperla brevis (Banks, 1895). 


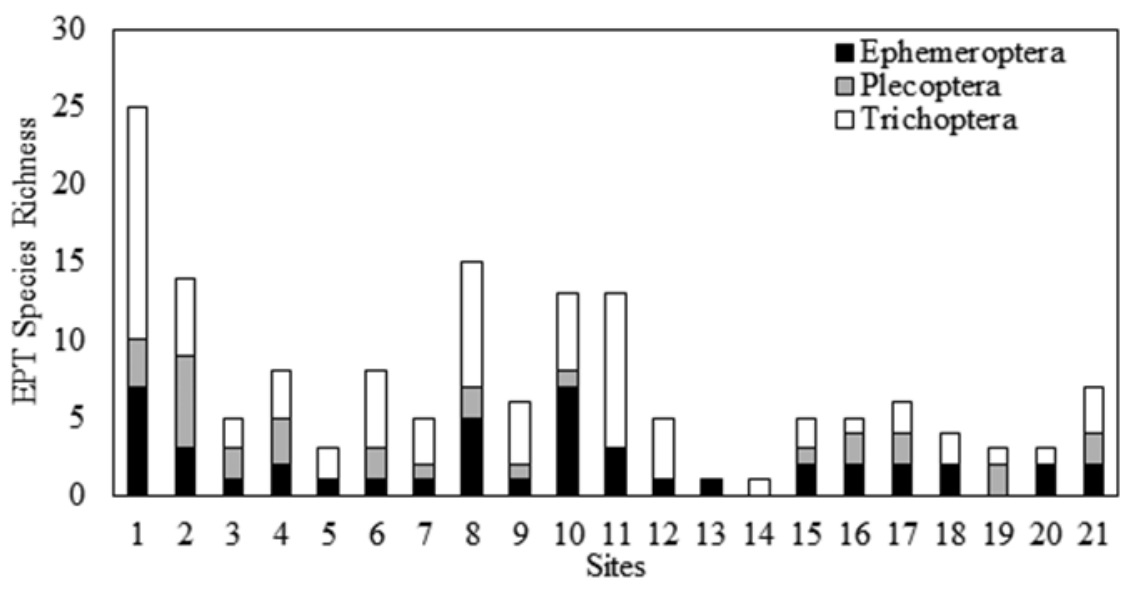

Figure 2. Ephemeroptera, Plecoptera, and Trichoptera species richness recovered from Isle Royale National Park sites during 2013. Site numbers correspond to those in Table 1.

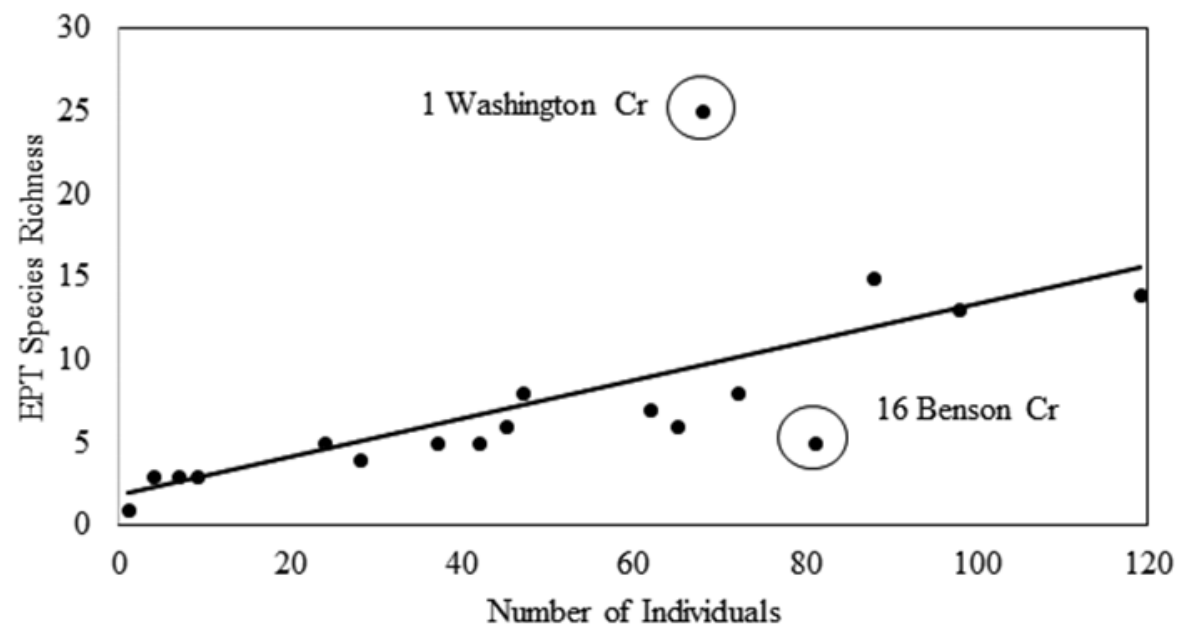

Figure 3. Relationship of Ephemeroptera, Plecoptera, and Trichoptera species richness to the number of individuals found at 18 sites where full samples were taken on Isle Royale National Park, 2013. Circled points indicate sites that had higher and lower than predicted richness. Diagonal is line-of-best-fit.

We were unable to produce reliable predictions of EPT species richness for ISRO with the number of complete samples at hand. Cumulative richness from complete samples yielded 68 species (Fig. 7). Five additional species from three incomplete samples bring the total to 73 species.

Size of ISRO EPT Species Versus Mainland. Mainland EPT were significantly different in size across orders (Kruskal-Wallis, $\mathrm{H}=13.9$, $\mathrm{df}=2, \mathrm{p}=0.0009$ ), with stoneflies having the largest average size at $11.13 \mathrm{~mm}$ (Fig. 8). Alternatively, EPT size on ISRO 


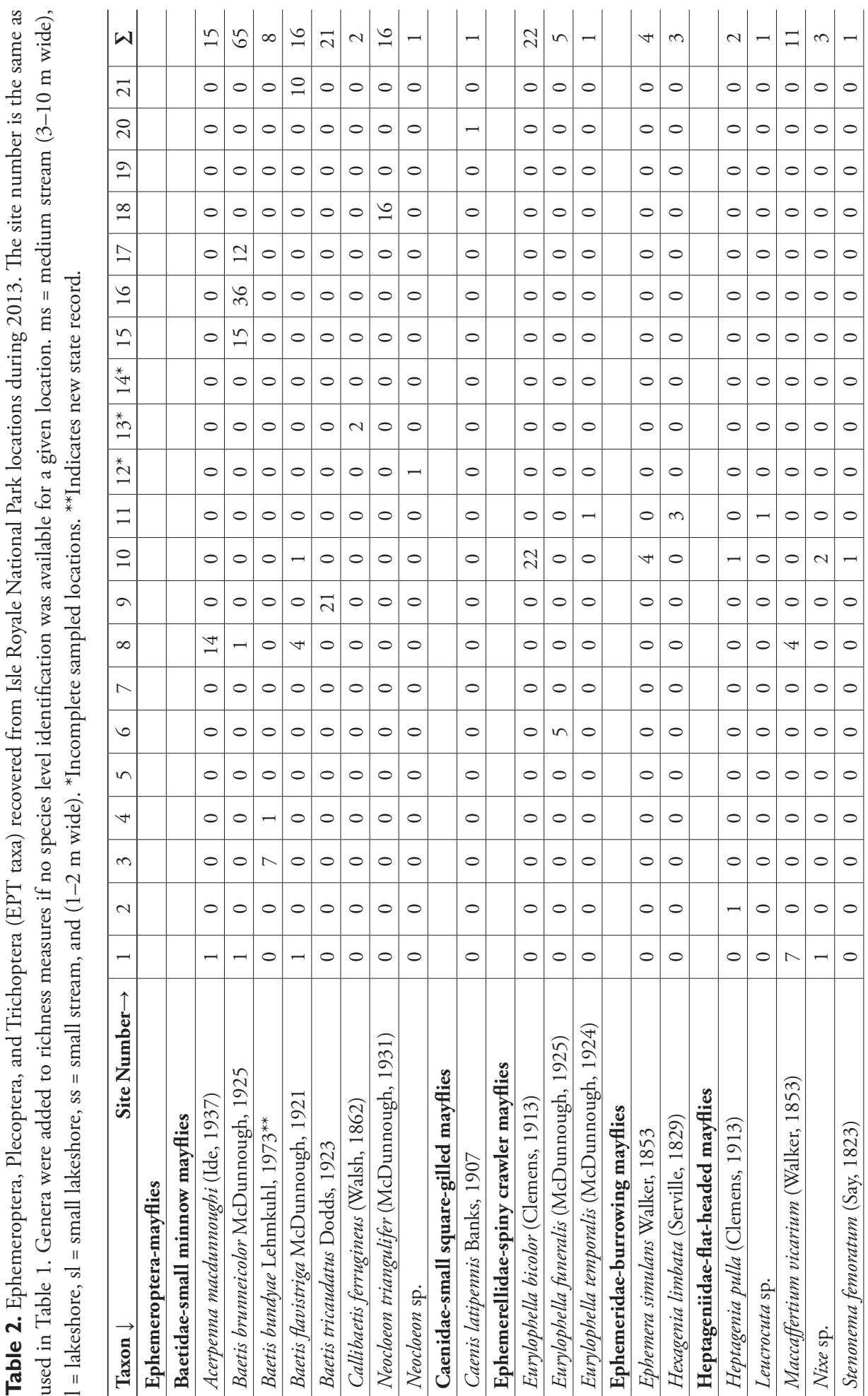




\begin{tabular}{|c|c|c|c|c|c|c|c|c|c|c|c|c|c|c|c|c|c|}
\hline $\mathbf{W}$ & 6 & $\beth$ & $\Xi$ & $n$ & $\bullet$ & $\hat{\lambda}$ & $\hat{\alpha}$ & ৫্ট & $\Re$ & 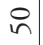 & $\Lambda$ & $\infty$ & - & $\cong$ & 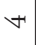 & $a$ & $=$ \\
\hline$v$ & $\psi$ & 0 & 0 & 0 & 0 & 0 & $\approx$ & 0 & 0 & 0 & 0 & - & 0 & $\cong$ & 0 & - & 0 \\
\hline$N$ & 0 & 0 & 0 & $\sim$ & 0 & 0 & 0 & 0 & 0 & 0 & 0 & 0 & 0 & 0 & 0 & 0 & 0 \\
\hline & 0 & 0 & 0 & 0 & 0 & 0 & - & 0 & 6 & 0 & 0 & 0 & 0 & 0 & 0 & 0 & 0 \\
\hline & 0 & 0 & $\wedge$ & 0 & 0 & 0 & 0 & 0 & 0 & 0 & 0 & 0 & 0 & 0 & 0 & 0 & 0 \\
\hline - & 0 & 0 & $\psi$ & 0 & 0 & 0 & 0 & $\stackrel{\infty}{-}$ & $\vec{\sim}$ & 0 & 0 & 0 & 0 & 0 & 0 & 0 & 0 \\
\hline$\exists$ & 0 & 0 & - & 0 & 0 & 0 & 0 & $\wedge$ & $n$ & 0 & 0 & 0 & 0 & 0 & 0 & 0 & 0 \\
\hline$\tilde{\partial}$ & 0 & 0 & $\sim$ & 0 & 0 & 0 & 0 & 0 & $\approx$ & 0 & 0 & 0 & 0 & 0 & 0 & 0 & 0 \\
\hline$t$ & 0 & 0 & 0 & 0 & 0 & 0 & 0 & 0 & 0 & 0 & 0 & 0 & 0 & 0 & 0 & 0 & 0 \\
\hline$\stackrel{*}{m}$ & 0 & 0 & 0 & 0 & 0 & 0 & 0 & 0 & 0 & 0 & 0 & 0 & 0 & 0 & 0 & 0 & 0 \\
\hline 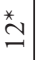 & 0 & 0 & 0 & 0 & 0 & 0 & 0 & 0 & 0 & 0 & 0 & 0 & 0 & 0 & 0 & 0 & 0 \\
\hline Z & 0 & 0 & 0 & 0 & 0 & 0 & 0 & 0 & 0 & 0 & 0 & 0 & 0 & 0 & 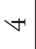 & 0 & 0 \\
\hline 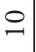 & - & 0 & 0 & 0 & 0 & 0 & $\vec{n}$ & 0 & 0 & 0 & 0 & 0 & 0 & $\sim$ & 0 & 0 & 0 \\
\hline$\lambda$ & 0 & 0 & 0 & 0 & 0 & 0 & 0 & - & 0 & 0 & 0 & 0 & 0 & 0 & 0 & $\psi$ & 0 \\
\hline & 0 & $=$ & 0 & 0 & 0 & 0 & 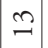 & - & 0 & 0 & 0 & 0 & 0 & 0 & 0 & 0 & $=$ \\
\hline & 0 & 0 & 0 & - & 0 & 0 & 0 & 0 & 0 & $\wedge$ & 0 & 0 & 0 & 0 & 0 & 0 & 0 \\
\hline & 0 & 0 & 0 & 0 & 0 & 0 & 0 & $\approx$ & 0 & $\beth$ & 0 & 0 & 0 & 0 & 0 & 0 & 0 \\
\hline & 0 & 0 & 0 & $\forall$ & 0 & 0 & 0 & 0 & 0 & 0 & 0 & 0 & 0 & 0 & 0 & 0 & 0 \\
\hline & 0 & 0 & 0 & ป & 0 & 0 & 0 & $\simeq$ & - & 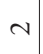 & 0 & 0 & 0 & 0 & 0 & 0 & 0 \\
\hline & 0 & 0 & 0 & 0 & 0 & 0 & - & 0 & 0 & $\beth$ & 0 & 0 & 0 & 0 & 0 & 0 & 0 \\
\hline & - & 0 & 0 & $n$ & $\bullet$ & $\curvearrowright$ & - & 0 & 0 & $\lambda$ & $\lambda$ & $\curvearrowright$ & 0 & 0 & 0 & - & 0 \\
\hline & 0 & 0 & 0 & - & 0 & 0 & $n$ & $\checkmark$ & 0 & 0 & 0 & 0 & - & 0 & 0 & $n$ & 0 \\
\hline
\end{tabular}

ลิ

点节

$\stackrel{*}{\stackrel{*}{2}}$

至

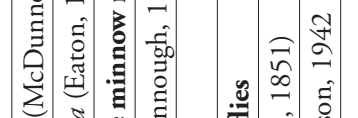

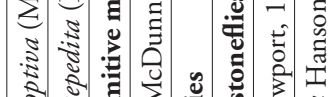

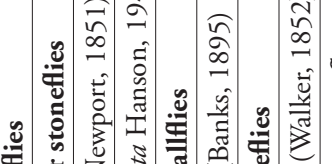

족

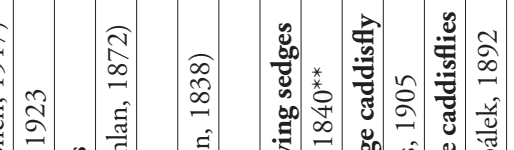

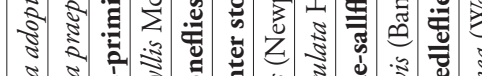

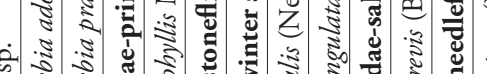

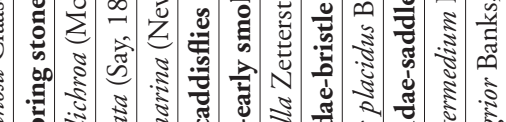
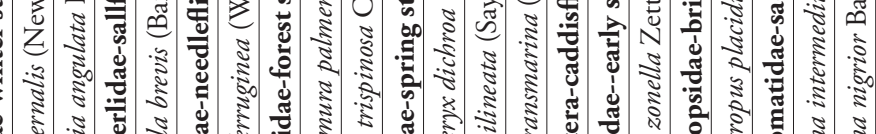


\begin{tabular}{|c|c|c|c|c|c|c|c|c|c|c|c|c|c|c|c|c|c|c|c|c|c|c|c|c|c|c|c|}
\hline $\mathbf{W}$ & & $\approx$ & 0 & $\stackrel{m}{=}$ & - & 6 & $\sim$ & 0 & - & $a$ & ㄱ. & & $m$ & 0 & - & & $\infty$ & $\vec{v}$ & & $\wedge 9$ & $\approx 1$ & 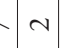 & - & - & & $--\rightarrow$ & $\sim$ \\
\hline a & & 0 & 0 & 0 & 0 & 6 & 0 & 0 & 0 & 0 & 0 & & 0 & 0 & 0 & & 0 & 0 & & 00 & 00 & 0 & 0 & 0 & & 0 & 0 \\
\hline iి & & 0 & 0 & 0 & 0 & 0 & 0 & 0 & 0 & 0 & 0 & & 0 & 0 & 0 & & 0 & 0 & & 00 & 00 & 0 & 0 & 0 & & \begin{tabular}{l|l|}
0 & -1 \\
\end{tabular} & 0 \\
\hline 2 & & 0 & 0 & 0 & 0 & 0 & 0 & 0 & 0 & 0 & 0 & & 0 & 0 & 0 & & 0 & 0 & & 00 & 00 & 0 & 0 & 0 & & $\begin{array}{lll}0 & 0\end{array}$ & 0 \\
\hline$\infty$ & & 0 & 0 & 0 & 0 & 0 & 0 & 0 & 0 & 0 & 0 & & 0 & 0 & 0 & & 0 & 0 & & 00 & 00 & 0 & 0 & 0 & & 0. & 0 \\
\hline 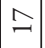 & & 0 & 0 & 0 & 0 & 0 & 0 & 0 & 0 & 0 & 0 & & 0 & 0 & 0 & & 0 & - & & 00 & 00 & 0 & 0 & 0 & & 0 & 0 \\
\hline$(0$ & & 0 & 0 & 0 & 0 & 0 & 0 & 0 & 0 & 0 & 0 & & 0 & 0 & 0 & & 0 & $N$ & & 00 & 00 & 0 & 0 & 0 & & 0 & 0 \\
\hline$\simeq$ & & 0 & 0 & 0 & 0 & 0 & 0 & 0 & 0 & 0 & 0 & & 0 & 0 & 0 & & 0 & 0 & & 00 & 00 & 00 & 0 & 0 & & 00 & $N$ \\
\hline 洁 & & 0 & 0 & 0 & 0 & 0 & 0 & 0 & 0 & 0 & 0 & & 0 & 0 & 0 & & 0 & 0 & & 00 & 00 & 00 & 0 & 0 & & -0 & 0 \\
\hline$\stackrel{*}{\stackrel{m}{=}}$ & & 0 & 0 & 0 & 0 & 0 & 0 & 0 & 0 & 0 & 0 & & 0 & 0 & 0 & & 0 & 0 & & 00 & 00 & 00 & 0 & 0 & & 0. & 0 \\
\hline$\stackrel{*}{=}$ & & 0 & 0 & 0 & 0 & 0 & 0 & 0 & 0 & 0 & 0 & & 0 & 0 & 0 & & 0 & 0 & & $\wedge \circ$ & 00 & $\sim \sim$ & $1-$ & 0 & & 00 & 0 \\
\hline$=$ & & $\approx$ & 0 & 0 & 0 & 0 & 0 & 0 & 0 & 0 & 0 & & 0 & 0 & 0 & & - & 0 & & 09 & $\beth 0$ & 0 & 0 & 0 & & 0.0 & 0 \\
\hline 으 & & $n$ & 0 & 0 & 0 & 0 & 0 & 0 & 0 & 0 & 0 & & 0 & 0 & 0 & & $\wedge$ & 0 & & 00 & 00 & 0 & 0 & 0 & & 0.0 & 0 \\
\hline 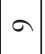 & & 0 & 0 & 0 & 0 & 0 & 0 & 0 & 0 & 0 & 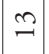 & & 0 & 0 & 0 & & 0 & 0 & & 00 & 00 & 00 & 0 & 0 & & 0. & 0 \\
\hline$\infty$ & & 0 & 0 & 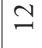 & 0 & 0 & $\sim$ & 0 & 0 & $n$ & 0 & & $\sim$ & 0 & 0 & & 0 & 0 & & 00 & 00 & 00 & 0 & 0 & & 0. & 0 \\
\hline N & & 0 & 0 & 0 & 0 & 0 & 0 & 0 & 0 & 0 & 0 & & 0 & 0 & 0 & & 0 & 0 & & 00 & 00 & 0 & 0 & 0 & & 0.0 & 0 \\
\hline$\bullet$ & & 0 & 0 & 0 & 0 & 0 & 0 & 0 & 0 & 0 & $\curvearrowright$ & & 0 & 0 & 0 & & 0 & $n$ & & 00 & 00 & 0 & 0 & 0 & & 00 & 0 \\
\hline $\ln$ & & 0 & 0 & 0 & 0 & 0 & 0 & 0 & 0 & 0 & 0 & & 0 & 0 & 0 & & 0 & 0 & & 00 & 00 & 0 & 0 & 0 & & 00 & 0 \\
\hline 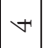 & & 0 & 0 & 0 & 0 & 0 & 0 & 0 & 0 & 0 & 0 & & 0 & 0 & 0 & & 0 & $n$ & & 00 & 00 & 0 & 0 & 0 & & 00 & 0 \\
\hline$n$ & & 0 & 0 & 0 & 0 & 0 & 0 & 0 & 0 & 0 & 0 & & 0 & 0 & 0 & & 0 & 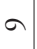 & & 00 & 00 & 0 & 0 & 0 & & 00 & 0 \\
\hline 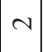 & & 0 & 0 & 0 & 0 & 0 & 0 & 0 & 0 & 0 & 0 & & 0 & 0 & 0 & & 0 & - & & 00 & 00 & 0 & 0 & 0 & & 00 & 0 \\
\hline- & & 0 & 0 & - & - & 0 & 0 & 0 & - & 6 & 0 & & - & 0 & - & & 0 & 0 & & 00 & 01 & 0 & 0 & - & & 00 & 0 \\
\hline 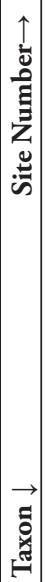 & 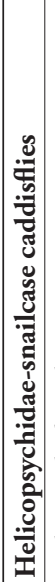 & 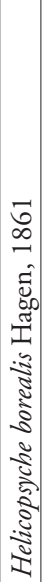 & 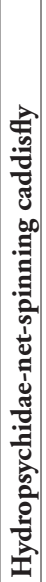 & 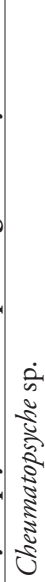 & 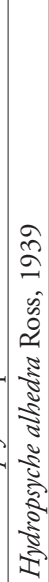 & 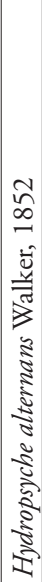 & 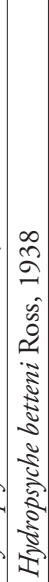 & 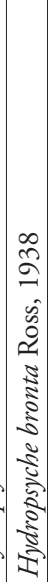 & 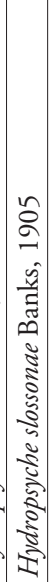 & 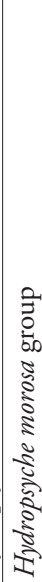 & 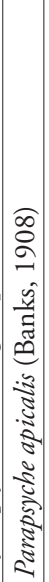 & 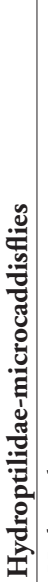 & 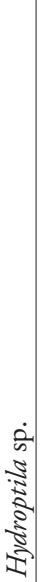 & 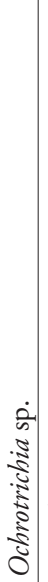 & 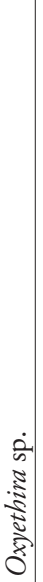 & 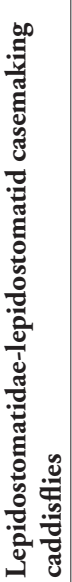 & 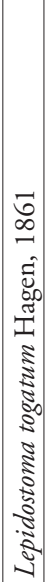 & 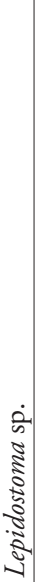 & 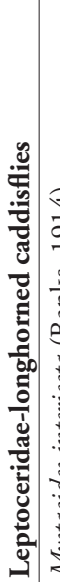 & 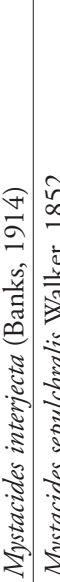 & 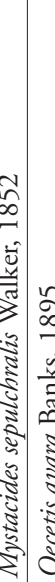 & 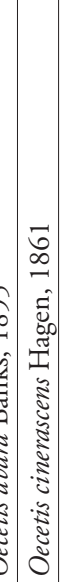 & 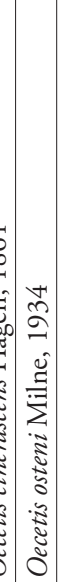 & 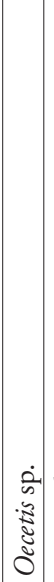 & 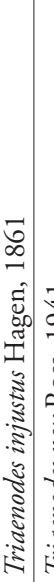 & 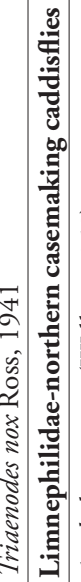 & 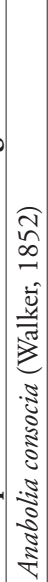 \\
\hline
\end{tabular}




\begin{tabular}{|c|c|c|c|c|c|c|c|c|c|c|c|c|c|c|c|c|c|c|c|c|c|c|}
\hline & - & 0 & $\exists$ & $\sim$ & $\sim$ & $\sim$ & $n$ & - & - & - & 6 & $\infty$ & $n$ & - & $\stackrel{0}{-1}$ & 6 & $\sim$ & - & ○ & - & 0 & $\underset{+}{\infty}$ \\
\hline $\mathrm{N}$ & 0 & 0 & 0 & 0 & 0 & 0 & 0 & 0 & 0 & 0 & 0 & 0 & 0 & 0 & 0 & 0 & 0 & 0 & 0 & 0 & 0 & 0 \\
\hline$\sqrt{ }$ & 0 & 0 & 0 & 0 & 0 & 0 & 0 & 0 & 0 & 0 & 0 & 0 & 0 & 0 & 0 & 0 & 0 & 0 & 0 & 0 & 0 & 0 \\
\hline 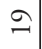 & 0 & 0 & 0 & 0 & $\sim$ & 0 & 0 & 0 & 0 & 0 & 0 & 0 & 0 & 0 & 0 & 0 & 0 & 0 & 0 & 0 & 0 & 0 \\
\hline 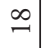 & 0 & $N$ & 0 & 0 & 0 & 0 & 0 & 0 & 0 & 0 & $n$ & 0 & 0 & 0 & 0 & 0 & 0 & 0 & 0 & 0 & 0 & 0 \\
\hline$=$ & 0 & 0 & 0 & 0 & 0 & 0 & 0 & 0 & 0 & 0 & 0 & 0 & 0 & 0 & $a$ & 0 & 0 & 0 & 0 & 0 & 0 & 0 \\
\hline$\underline{-1}$ & 0 & 0 & 0 & 0 & 0 & 0 & 0 & 0 & 0 & 0 & 0 & 0 & 0 & 0 & 0 & 0 & 0 & 0 & 0 & 0 & 0 & 0 \\
\hline$\theta$ & 0 & 0 & 0 & 0 & 0 & 0 & 0 & 0 & 0 & 0 & 0 & 0 & 0 & 0 & 0 & 0 & 0 & 0 & 0 & 0 & 0 & 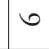 \\
\hline \pm & 0 & 0 & 0 & 0 & 0 & 0 & 0 & 0 & 0 & 0 & 0 & 0 & 0 & 0 & 0 & 0 & 0 & 0 & 0 & 0 & 0 & 0 \\
\hline$n$ & 0 & 0 & 0 & 0 & 0 & 0 & 0 & 0 & 0 & 0 & 0 & 0 & 0 & 0 & 0 & 0 & 0 & 0 & 0 & 0 & 0 & 0 \\
\hline 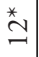 & 0 & - & 0 & 0 & 0 & 0 & 0 & 0 & 0 & 0 & 0 & 0 & 0 & 0 & 0 & 0 & 0 & 0 & 0 & 0 & 0 & 0 \\
\hline 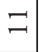 & 0 & $\sim$ & 0 & 0 & 0 & 0 & 0 & - & 0 & 0 & 0 & $\infty$ & $n$ & 0 & 0 & 6 & 0 & - & 0 & 0 & 0 & 0 \\
\hline 0 & 0 & 0 & 0 & 0 & 0 & 0 & 0 & 0 & - & 0 & 0 & 0 & 0 & 0 & 0 & 0 & 0 & 0 & 0 & 0 & 0 & $\sim$ \\
\hline$a$ & 0 & 0 & 0 & 0 & 0 & 0 & 0 & 0 & 0 & 0 & 0 & 0 & 0 & 0 & - & 0 & 0 & 0 & 0 & 0 & 0 & $n$ \\
\hline$\infty$ & 0 & 0 & 0 & 0 & 0 & 0 & 0 & 0 & 0 & - & 0 & 0 & 0 & 0 & 0 & 0 & 0 & 0 & 0 & 0 & 6 & $m$ \\
\hline & 0 & 0 & in & 0 & 0 & 0 & 0 & 0 & 0 & 0 & 0 & 0 & 0 & 0 & 0 & 0 & 0 & 0 & 0 & 0 & $\sim$ & $\sigma$ \\
\hline 0 & 0 & 0 & 6 & 0 & 0 & 0 & 0 & 0 & 0 & 0 & 0 & 0 & 0 & 0 & 0 & 0 & 0 & 0 & -1 & 0 & 0 & $\stackrel{-}{1}$ \\
\hline$n$ & 0 & 0 & 0 & $\sim$ & 0 & 0 & - & 0 & 0 & 0 & 0 & 0 & 0 & 0 & 0 & 0 & 0 & 0 & 0 & 0 & 0 & 0 \\
\hline 梖 & - & 0 & 0 & 0 & 0 & 0 & 0 & 0 & 0 & 0 & 0 & 0 & 0 & 0 & 0 & 0 & 0 & 0 & $n$ & 0 & 0 & 0 \\
\hline$m$ & 0 & 0 & 0 & 0 & 0 & 0 & 0 & 0 & 0 & 0 & 0 & 0 & 0 & 0 & 0 & 0 & 0 & 0 & 0 & 0 & 0 & $\infty$ \\
\hline & 0 & 0 & 0 & 0 & 0 & $N$ & 0 & 0 & 0 & 0 & 0 & 0 & 0 & 0 & 0 & 0 & 0 & 0 & $v$ & 0 & 0 & - \\
\hline & 0 & & 0 & 0 & 0 & 0 & 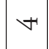 & 0 & 0 & 0 & $m$ & 0 & 0 & - & 0 & 0 & $\sim$ & 0 & 0 & - & $\sim$ & $\psi$ \\
\hline
\end{tabular}




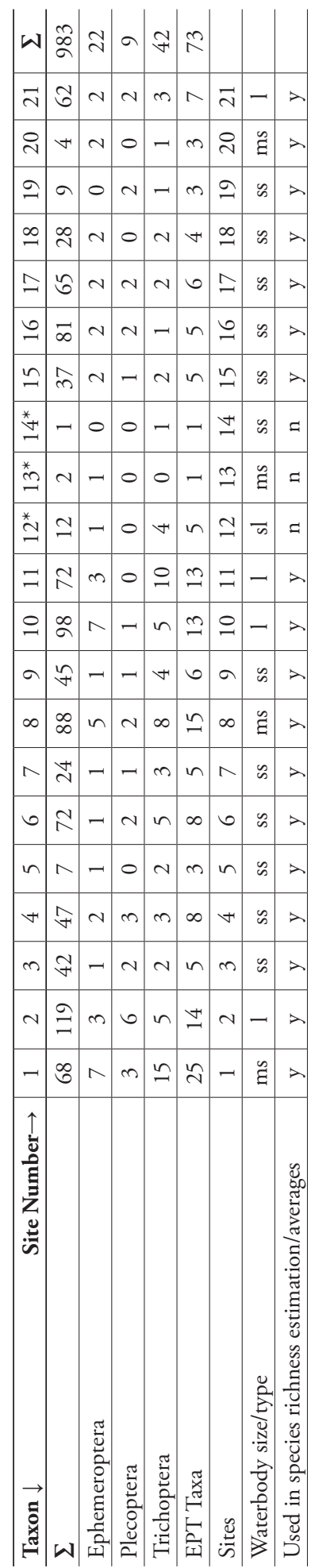




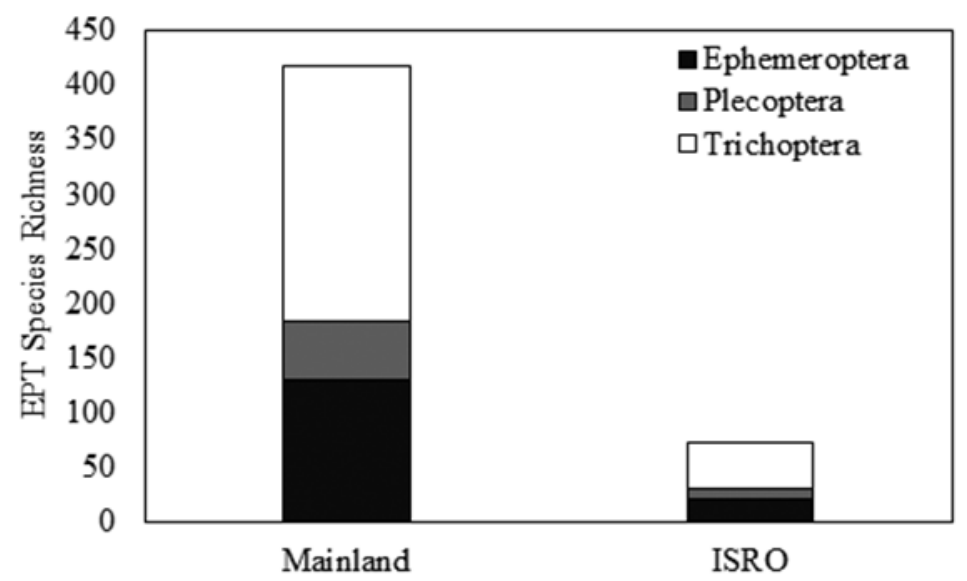

Figure 4. Comparison of Ephemeroptera, Plecoptera, and Trichoptera species richness for mainland around Lake Superior versus that found on Isle Royale National Park sites during 2013.

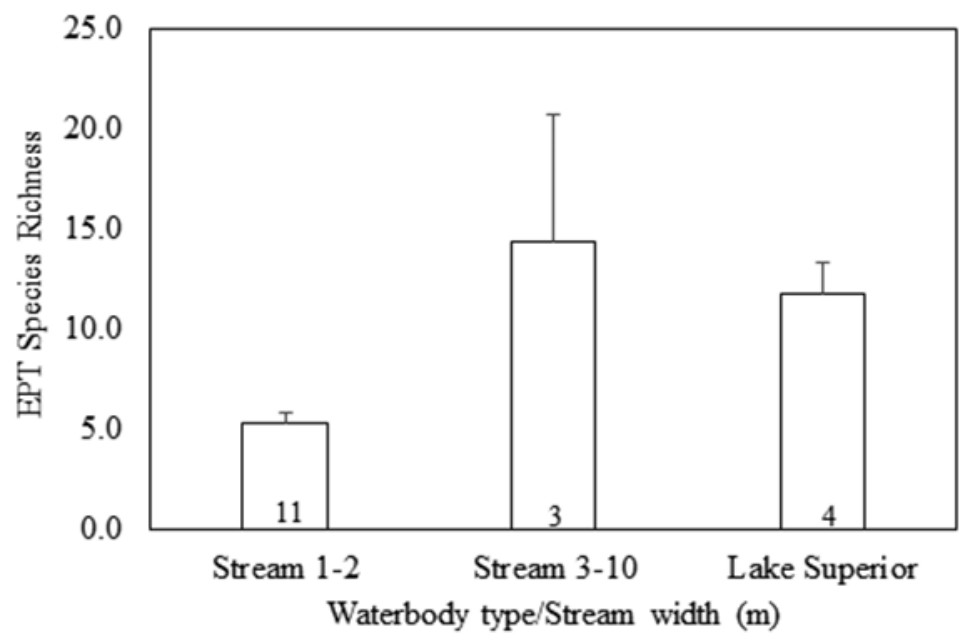

Figure 5. Mean site Ephemeroptera, Plecoptera, and Trichoptera species richness by waterbody type and stream size. Sites represented by incomplete samples excluded. Numbers in columns indicate number of sites. Error bars indicated standard error of the mean.

was not significantly different across orders, but the margin was close with the mean size of caddisflies being somewhat larger than other orders (Kruskal-Wallis, $\mathrm{H}=5.7$, $\mathrm{df}=2, \mathrm{p}=0.059$ ) (Fig. 8). With respect to comparisons between sources, mayfly species size between the mainland and ISRO was not significantly different (Mann-Whitney U-test, $\mathrm{U}=1403.0, \mathrm{P}(1)=0.44, \mathrm{P}(2)=0.89)$. A frequency histogram demonstrates that sizes of mayflies overlapped greatly for mainland and ISRO sources (Fig. 9). Stonefly species were significantly smaller on ISRO than they were on the mainland (Mann- 


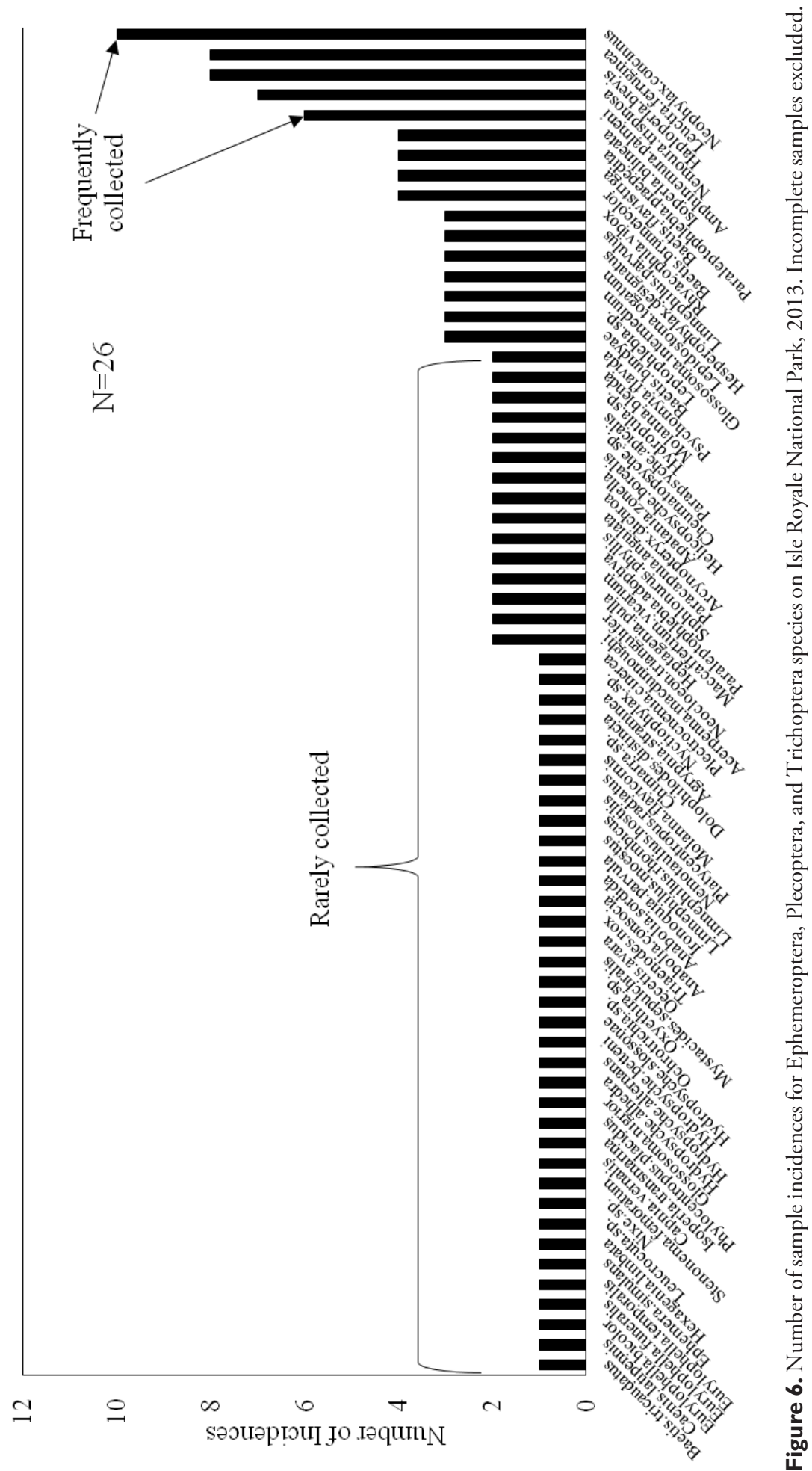




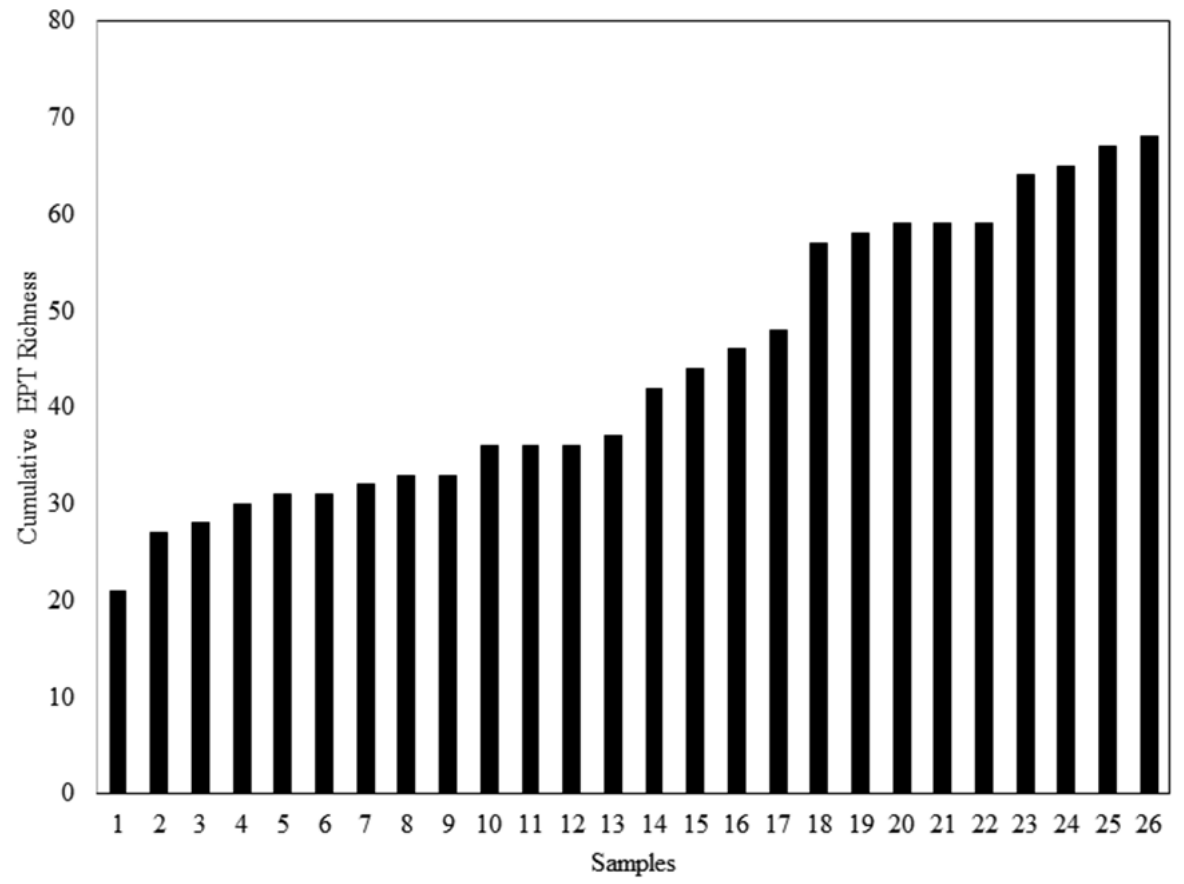

Figure 7. Cumulative Ephemeroptera, Plecoptera, and Trichoptera species richness generated from 26 complete samples collected from Isle Royale National Park, 2013.

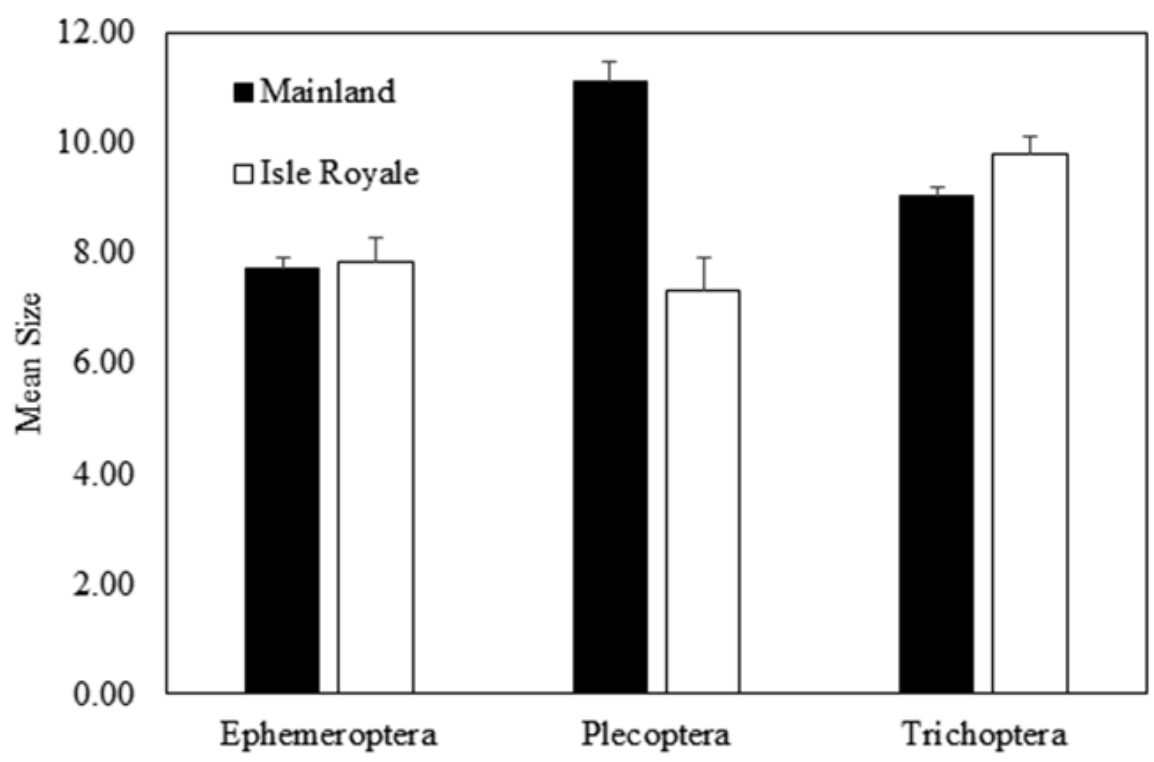

Figure 8. Mean and standard error of minimum size of Ephemeroptera, Plecoptera, and Trichoptera for species inhabiting the Lake Superior mainland and species sampled from Isle Royale National Park lakeshore and streams. 

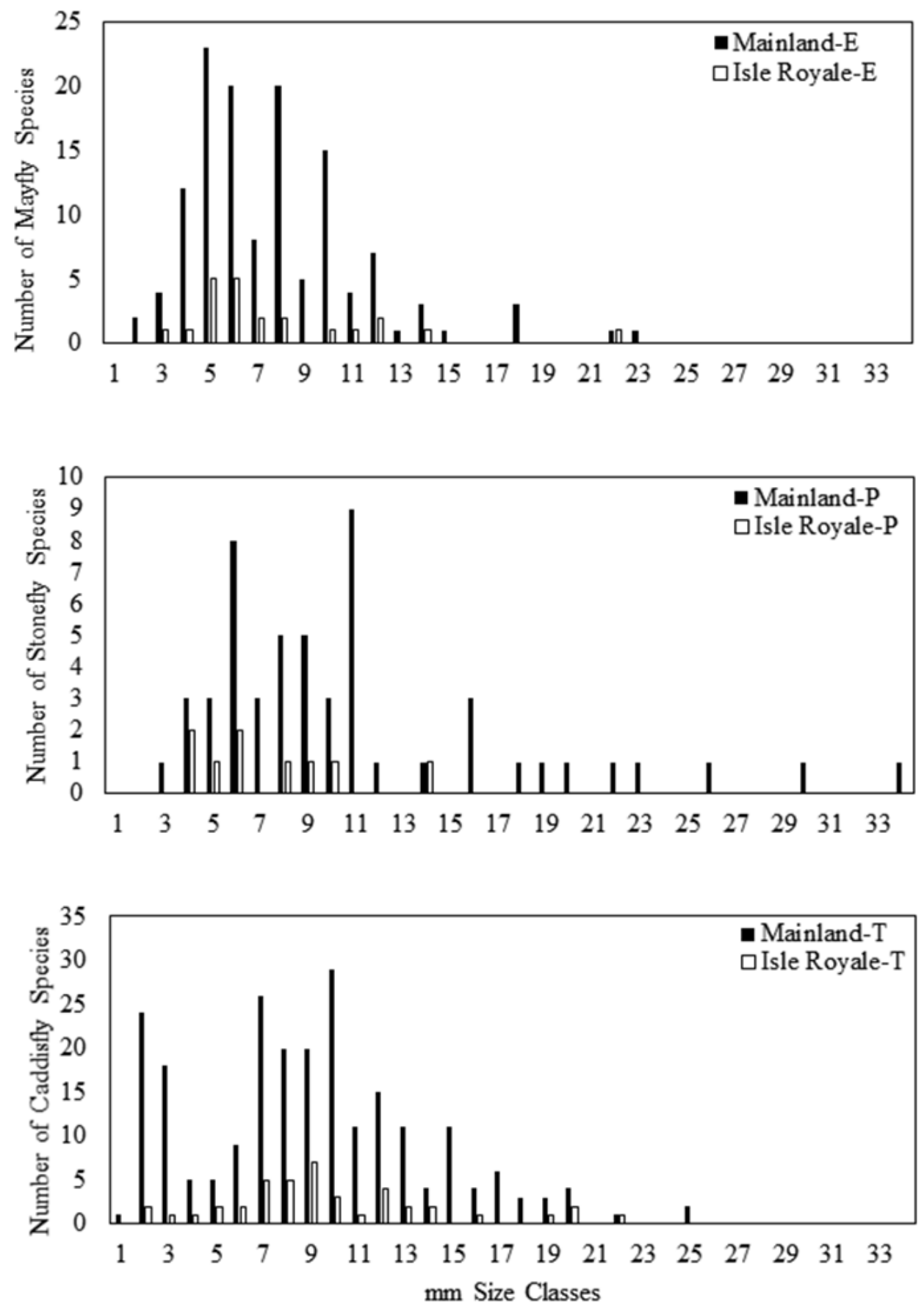

Figure 9. Size frequency histogram of Ephemeroptera (E), Plecoptera (P), and Trichoptera $(\mathbf{T})$ species inhabiting Lake Superior mainland and species sampled from Isle Royale National Park lakeshore and streams. 
Whitney $\mathrm{U}$-test, $\mathrm{U}=149.5, \mathrm{P}(1)=0.034, \mathrm{P}(2)=0.067)$. Mainland stonefly species ranged 3-34 mm in size, while on ISRO, they ranged 4-14 mm (Fig. 9). Caddisfly species size was not significantly different between sources (Mann-Whitney U-test, U=5231.0, $\mathrm{P}(1)=0.239, \mathrm{P}(2)=0.478)$ with the size distribution of the two sources being nearly identical (Fig. 9).

\section{Discussion}

Aquatic resources on ISRO, with the current state of sampling, support only $17.5 \%$ of the EPT species found on the mainland surrounding Lake Superior (Fig. 4). Since the number of species found in only one or two samples is large (Fig. 6) and the accumulation of species has a steep curve (Fig. 7), we assume that this proportion will rise with greater effort. However, we still expect that $<50 \%$ of the mainland species will be recovered from ISRO. Caddisflies seemed to be the most effective colonizers of the island, their observed richness being nearly $57.6 \%$ of the EPT species recorded to date (Fig. 4). On the other hand, stoneflies seem to be ill suited for traversing the distance from the mainland to ISRO. However, those that colonized ISRO often occurred at many sites (Fig. 6) and often in high abundance.

Small streams on ISRO supported low EPT species richness, while the number of species climbed dramatically in larger streams (Fig. 5). It is probable that many of these small streams freeze into the streambed during the winter, leaving only a few hardy species with egg diapause to overwinter. The larger, species rich streams must continue flowing during winter. Caddisflies and mayflies were most responsible for the increase in species richness of larger streams. Interestingly, Washington and Grace creeks, though being of nearly identical size and gradient, and being geographically close, were quite different in assemblage composition with a Sorensen's Index of Similarity being only $38 \%$.

The shores of Lake Superior supported a moderate diversity of EPT with low variability in the number of species. The communities varied greatly between cold beaches exposed to the fetch of the lake and the protected bays. Exposed lake shores produced many more stoneflies than protected bays, presumably due to the colder water. Caddisflies and mayflies were much more species rich in the protected bays.

Body Size as a Predictor of ISRO EPT. Others have studied the evolution of body size of vertebrates on isolated islands as a function of food quality, island area, and interactions with other species (Boyer and Jetz 2010). We are focusing on size as a factor important only in the initial colonization of aquatic insects to ISRO. We suggest that large species may not colonize ISRO successfully and that small species would have an advantage since they could use updrafts from Minnesota, Ontario, or the Keweenaw Peninsula of Michigan to reach ISRO. We have found that the assemblage of stoneflies on ISRO support this hypothesis since they were significantly smaller than on the mainland (Fig. 9). The two largest stonefly species on ISRO, I. bilineata (Say, 
1824) (9-9.5 mm) and A. dichroa (McLachlan, 1872) (14-15 mm), occurred in the lake and presumably have used it to colonize the island. Stoneflies are often considered poor fliers (Stewart and Stark 2002), although there is little direct evidence for this. Malmqvist (2000) found that wing length was positively related to range size and that species with short wings were most likely to be rare and isolated on the landscape. One the mainland, 10-20 large species in the families Perlodidae, Perlidae, and Pteronarcyidae may be present in the same stream. Stoll et al. (2014) found that the presence of fish in the regional species pool was a most important determiner of colonization of restored stream reaches. We suggest that the distance and disruption of normal habitat, e.g. the lake, for larval and adult stonefly species limits most large species from reaching ISRO. One mechanism for limiting flight of large stoneflies is that they fly with the body inclined at a $25-45^{\circ}$ angle, conferring considerable drag during flight (DeWalt pers. obs.). The complete absence of truly large stoneflies on ISRO suggests that larger stonefly species do not have the energy reserves or aerodynamics to fly or draft the long distances from the mainland to ISRO.

Mayflies and caddisflies do not support the hypothesis that smaller species are more likely to colonize ISRO (Figs 8, 9). Mayfly wing length has been demonstrated to be positively related to range size, a trait that would increase the possibility of them flying from mainland to ISRO (Malmqvist 2000). Mayflies and caddisflies are generally thought of as stronger fliers than stoneflies. Indeed, anecdotal evidence from weather radar supports the idea that large, burrowing mayflies in the genus Hexagenia (Ephemeridae) fly considerable distances equivalent to that that isolates ISRO from the mainland (Washington Post 2014). The body axis orientation of mayflies and caddisflies is more horizontal during flight; presumably, more power is transferred to forward motion without the drag that stoneflies endure. In addition, an unknown number of mayfly and caddisfly species included in the ISRO taxa list certainly occur within Lake Superior. This would make the habitat from mainland to ISRO more continuous and allow more species of both orders to reach the island. Of course, examination of many more species traits is necessary to determine which factors are most important to the postglacial recolonization of ISRO by all three groups.

Taxa of Significance. Most species reported herein have never been reported in the literature from the ISRO and represent a leap in knowledge of the species of aquatic insects that inhabit the park. Some species were of particular interest because of their rarity in the region, their being new state records, or because they have been known in the region under different names until recently. We present an annotated list of those species.

\section{Ephemeroptera}

Acerpenna macdunnoughi (Ide, 1937). Several records exist for small streams in Marquette County, Michigan (Randolph and McCafferty 1998) and nowhere else in the state. It was found in both Washington (Site 1) and Grace creeks (Site 8), the latter in abundance. 
Baetis bundyae Lehmkuhl, 1973. This boreal/tundra species has not been reported from Michigan before (Randolph and McCafferty 1998), but is known from nearby northeastern Minnesota (Lager et al. 1982). This coldwater species was found in a two locations: Huginnin Creek at Huginnin Cove (Site 3) and at a nearby unnamed tributary to Lake Superior (Site 4). This area is kept cold by the lake breezes, producing a southern refuge for the species.

Callibaetis ferrugineus (Walsh, 1862). This is the first record of the species for upper Michigan (Randolph and McCafferty 1998). Two nymphs were taken from the outlet of Lake Richie (Site 12) along the Indian Portage Trail.

Neocloeon triangulifer (McDunnough, 1931). This species, under the name Centroptilum triangulifer (McDunnough, 1931), was only recently added to the Michigan mayfly list, the new records being from Baraga and Marquette counties in the Upper Peninsula (McCafferty 2009). Jacobus and Wiersema (2014) recently moved this species to Neocloeon. A large population was taken from the flooded mouth of a small tributary to Lake Superior near Moskey Basin Campground (Site 18).

Paraleptophlebia praepedita (Eaton, 1884). This has been rarely collected from Michigan, and only in the lower third of the state (Randolph and McCafferty 1998). It was taken from several tributaries to Moskey Basin (Sites 15-18). This is the first record of the species in northern Michigan.

Siphlonurus phyllis McDunnough, 1923. This species has never been reported from the state (Randolph and McCafferty 1998). Its presence represents a new state record.

\section{Plecoptera}

Capnia vernalis (Newport, 1851). This species is rare in the region. It was found only at the Lake Superior shoreline at Huginnin Cove (Site 2).

Amphinemura palmeni (Koponen, 1917). It is not surprising that this species was found on ISRO. We have listed it here to call attention to a relatively recent synonymy that has occurred. Probably hundreds of specimens exist in North American collections using the name $A$. linda (Ricker, 1952), a junior synonym (Boumans and Baumann 2012). This is apparently the only Amphinemura on the island and was found at five small streams (Sites 4, 15-17, 19).

Arcynopteryx dichroa (McLachlan, 1872). This Holarctic species is another rarity, being known only from the shores of Lake Superior in the region (Grubbs and Bright 2001). Until recently it was known as $A$. compacta (McLachlan, 1872), but all Nearctic specimens under that name are now referable to $A$. dichroa (Teslenko 2012).

\section{Trichoptera}

Apatania zonella Zetterstedt, 1840. This is a new state record for Michigan. Leonard and Leonard (1949) did not report it for the state, but it has been collected from 
Lake Superior shores in nearby Minnesota. We collected it from shoreline samples at Daisy Farm Campground (Site 10) and Rock Harbor (Site 21).

Ironoquia parvula Banks, 1900. This too is a new state record for Michigan. We collected two of the distinctive (Flint 1960) larvae from a small, white cedar swamp (site 5).

\section{Acknowledgments}

We wish to thank Ryan Maki of the National Park Service, Isle Royale National Park for permits to sample ISRO and for agreeing to wave fees to enter the park. We also thank the following colleagues for providing unpublished data from which to develop the EPT regional species pool available to ISRO for colonization: Dr. Tomas Klubertanz, University of Wisconsin-Rock County; Dr. David Houghton, Hillsdale College, Hillsdale Michigan; Dr. Patrick Randolph, University of California, Davis. The compilation of specimen databases was partially funded by the following grants: USA NSF DEB 09-18805 ARRA and a USA DOI-USFWS X-1-R-1.

\section{References}

Bick K, Janke RA, Linn RM, Peterson RO, Rutkowski D, Stottlemyer JR (1985) Isle Royale Biosphere Reserve: history of scientific studies. U.S. Man and Biosphere Program, U.S. MAB Report No. 11, Volume I. U.S. Department of the Interior, National Park Service, Science Publications Office, 115 pp.

Blahnik RJ, Holzenthal RW (2014) Review and redescription of species in the Oecetis avara group, with the description of 15 new species (Trichoptera, Leptoceridae). ZooKeys 376: 1-83. doi: 10.3897/zookeys.376.6047

Boumans L, Baumann RW (2012) Amphinemura palmeni is a valid Holarctic stonefly species (Plecoptera: Nemouridae). Zootaxa 3537: 59-75. http://www.mapress.com/ zootaxa/2012/f/zt03537p075.pdf

Bowden RD (1981) Benthic macroinvertebrates and chemistry of three streams on Isle Royale National Park, Michigan. Thesis. Michigan Technological University, Houghton, Michigan. Boyer AG, Jetz W (2010) Biogeography of body size in Pacific island birds. Ecography 33: 369-379. doi: 10.1111/j.1600-0587.2010.06315.x

Burks BD (1953) The mayflies or Ephemeroptera of Illinois. Bulletin of the Illinois Natural History Survey 26: 1-216.

Cao Y, DeWalt RE, Robinson JL, Tweddale T, Hinz L, Pessino M (2013) Using Maxent to model the historic distributions of stonefly species in Illinois streams and rivers: the effects of regularization and threshold selections. Ecological Modelling 259: 30-39. doi: 10.1016/j.ecolmodel.2013.03.012

DeWalt RE, Grubbs SA (2011) Updates to the stonefly Fauna of Illinois and Indiana. Illiesia 7: 31-50. http://www2.pms-lj.si/illiesia/papers/Illiesia07-03.pdf 
DeWalt RE, Favret C, Webb DW (2005) Just how imperiled are aquatic insects? A case study of stoneflies (Plecoptera) in Illinois. Annals of the Entomological Society of America 98: 941-950. doi: 10.1603/0013-8746(2005)098[0941:JHIAAI]2.0.CO;2

DeWalt RE, Cao Y, Tweddale T, Grubbs SA, Hinz L, Pessino M (2012) Ohio USA stoneflies (Insecta, Plecoptera): species richness estimation, distribution of functional niche traits, drainage affiliations, and relationships to other states. ZooKeys 178: 1-26. doi: 10.3897/ zookeys.178.2616

Flint OS Jr (1960) Taxonomy and biology of Nearctic limnephilid larvae (Trichoptera) with special reference to species in eastern United States. Entomologica Americana 40: 1-117.

Frison TH (1935) The stoneflies, or Plecoptera, of Illinois. Bulletin Illinois Natural History Survey 20: 281-467.

Grubbs SA, Bright E (2001) Arcynopteryx compacta (Plecoptera: Perlodidae), a Holarctic stonefly confirmed from Lake Superior, with a review and first checklist of the stoneflies of Michigan. The Great Lakes Entomologist 34: 77-84.

Grubbs SA, Pessino M, DeWalt RE (2013) Michigan Plecoptera (Stoneflies): distribution patterns and an updated species list. Illiesia 8: 162-173. http://www2.pms-lj.si/illiesia/papers/ Illiesia08-18.pdf

Houghton DC (2012) Biological diversity of the Minnesota caddisflies (Insecta, Trichoptera). ZooKeys 189: 1-389. doi: 10.3897/zookeys.189.2043

Jacobus LM, Wiersema NA (2014) The genera Anafroptilum Kluge, 2011 and Neocloeon Traver, 1932, reinstated status, in North America, with remarks about the global composition of Centroptilum Eaton, 1869 (Ephemeroptera: Baetidae). Zootaxa 3814(3): 385-391. doi: 10.11646/zootaxa.3814.3.5

Johnson DK (1980) Ecological relationships of aquatic macroinvertebrates in Siskiwit River, Isle Royale National Park, Michigan. Thesis. Michigan Technological University, Houghton, Michigan.

Kraft GJ, Mechenich DJ, Mechenich C, Cook JE, Seiler SM (2010) Assessment of natural resource conditions: Isle Royale National Park. Natural Resource Report NPS/NRPC/ WRD/NRR-2010/237, 219 pp.

Lager TM, Johnson MD, McCafferty WP (1982) The mayflies of northeastern Minnesota (Ephemeroptera). Proceedings of the Entomological Society of Washington 84: 729-741. Leonard JW, Leonard FA (1949) An annotated list of Michigan Trichoptera. Occasional Papers of the Museum of Zoology 522: 1-35. http://deepblue.lib.umich.edu/bitstream/2027.42/56960/1/OP522.pdf

Lowry R (2015) VassarStats: Website for Statistical Computation. http://vassarstats.net/ [accessed on 2 September 2015]

Malmqvist B (2000) How does wing length relate to distribution patterns of stoneflies (Plecoptera) and mayflies (Ephemeroptera)? Biological Conservation 93: 271-276. doi: 10.1016/ S0006-3207(99)00139-1

McCafferty WP (2009) New state and provincial North American records for 100 Ephemeroptera species. Transactions of the American Entomological Society 135(3): 353-368. doi: $10.3157 / 061.135 .0302$ 
Randolph RP, McCafferty WP (1998) Diversity and distribution of the mayflies (Ephemeroptera) of Illinois, Indiana, Kentucky, Michigan, Ohio, and Wisconsin. Ohio Biological Survey Bulletin New Series 13: 1-188.

Ross HH (1944) The caddisflies or Trichoptera of Illinois. Bulletin of the Illinois Natural History Survey 23: 1-326.

Sokal RR, Rohlf FJ (1981) Biometry, Principles and Practice of Statistics in Biological Research, $2^{\text {nd }}$ edition. W. H. Freeman and Company, New York, 859 pp.

Stewart KW, Stark BP (2002) Nymphs of North American Stonefly Genera, 2nd ed. Caddis Press, Columbus, Ohio, 510 pp.

Stoll S, Kail J, Lorenz AW, Sundermann A, Haase P (2014) The importance of the regional species pool, ecological species traits and local habitat conditions for the colonization of restored river reaches by fish. PLoS ONE 9(1): e84741. doi: 10.1371/journal.pone.0084741

Sun L, McCafferty WP (2008) Cladistics, classification and identification of the brachycercine mayflies (Insecta: Ephemeroptera: Caenidae). Zootaxa 1801: 1-239. http://www.mapress. com/zootaxa/2008/f/z01801p239f.pdf

Teslenko VA (2012) A taxonomic revision of the genus Arcynopteryx Klapálek, 1904 (Plecoptera, Perlodidae). Zootaxa 3329: 1-18. http://www.mapress.com/zootaxa/2012/f/ z03329p018f.pdf

Toczydlowski DG, Abramson T, Burdett RS (1979) Aquatic baseline on Isle Royale, Michigan. Technical Report Michigan Technological University, 72 pp.

Walker F (1852) Catalogue of the specimens of neuropterous insects in the collection of the British Museum 1: 1-192.

Washington Post (2014) Watch swarms of Mississippi River mayflies so thick they showed up on radar like a storm. 26 July 2014. http://www.washingtonpost.com/news/morning-mix/ wp/2014/07/28/wacth-swarms-of-mississippi-river-mayflies-so-thick-they-showed-up-onradar/ [accessed on 4 September 2015]

\section{Supplementary material I}

\section{Table S1. Raw specimen data in the form of an Excel comma delimited file} Authors: R. Edward DeWalt, Eric. J. South

Data type: occurence

Copyright notice: This dataset is made available under the Open Database License (http://opendatacommons.org/licenses/odbl/1.0/). The Open Database License $(\mathrm{ODbL})$ is a license agreement intended to allow users to freely share, modify, and use this Dataset while maintaining this same freedom for others, provided that the original source and author(s) are credited. 